\section{Marina Gets}

\section{Meinungsäußerungs- und Informations- freiheit im Internet aus der Sicht des Völkerrechts}

Berlin: Berlin Verlag Arno Spitz, 2002. - 200 S.

(Zugl.: Jena, Univ., Diss., 2001)

ISBN 3-8305-0032-7

Die Entwicklung des Internets als von seiner technischen Anlage her weitgehend grenzenloses globales Kommunikationsmittel hat zahlreiche rechtliche und gesellschaftspolitische Herausforderungen mit sich gebracht. Diese beziehen sich u. a. auf zivilrechtliche Fragen im Zusammenhang mit dem Stichwort „E-Commerce", auf strafrechtliche Probleme mit Blick auf z. B. rassistische Propaganda und auf öffentlich-rechtliche Aspekte präventiven staatlichen Handelns zur Gefahrenabwehr. Hierzu liegen bereits zahlreiche einschlägige Zeitschriftenaufsätze, Dissertationen und erste $\mathrm{Ha}-$ bilitationsschriften vor. Es fällt hingegen auf, dass die dem Internet und insgesamt den digitalen Kommunikations- und Informationsmedien inhärente globale Dimension zwar natürlich regelmäßig im wissenschaftlichen Schrifttum hervorgehoben wird, die daraus folgenden rechtlichen Konsequenzen aber oftmals nicht umfassend analysiert werden. Insofern ist bis heute ein Forschungsdefizit im Hinblick auf die eigentlichen internationalrechtlichen oder völkerrechtlichen Fragestellungen der modernen Informations- und Kommunikationsmedien zu konstatieren. Umso erfreulicher ist es, dass Martina Gets sich in ihrer von Karl M. Meessen betreuten Dissertation, die im Sommer 2001 von der Juristischen Fakultät der Universität Jena angenommen wurde, diesem Themenbereich widmet. Allerdings gelingt es Gets leider nicht, das einschlägige Schrifttum in ansprechendem Umfang aufzuarbeiten und $\mathrm{zu}$ den eigentlichen völkerrechtlichen Fragen des Internets vorzudringen.

Gets gliedert ihre Arbeit in drei Teile. Im ersten Teil geht es ihr im ersten Untersuchungsabschnitt zunächst um die Begriffe „Meinungsäußerungs- und Informationsfreiheit". Hierbei wird maßgeblich auf die deutsche Grundrechtsdogmatik eingegangen, um eine begriffliche Klärung herbeizuführen. Auch mit Blick auf das zitierte Schrifttum wird fast ausschließlich auf einschlägige Abhandlungen des deutschen Staats- und Verwaltungsrechts abgestellt.
Es finden sich keine Hinweise z. B. auf die Rechtsprechung des Europäischen Gerichtshofs für Menschenrechte (EuGHMR) oder die einschlägige Praxis im Rahmen des IPBürgR. Ebenso wenig wird auf die Praxis u. a. in der UNESCO, der ITU und der Generalversammlung der Vereinten Nationen eingegangen. $\mathrm{Da}-$ her bleibt es bei einer rechtlichen Begrifflichkeit, die zwar für das deutsche öffentliche Recht zutreffend ist, einen völkerrechtlichen Anspruch aber nicht erheben kann. Das kann für eine völkerrechtliche Arbeit nicht überzeugen. Zugleich fällt schon auf den ersten Seiten auf, dass Gets sich kaum mit dem einschlägigen völkerrechtlichen Schrifttum auseinander gesetzt hat. Das zeigt sich z. B. daran, dass die bis heute grundlegende Schrift von Alexandrowicz (The Law of Global Communications, New York u. a. 1971) nicht zitiert und verarbeitet wurde.

In den nächsten beiden Untersuchungsabschnitten des ersten Teils geht Gets dann ausführlich auf die technischen und sozialen Voraussetzungen der Meinungsäußerungs- und Informationsfreiheit im Internet sowie diesbezügliche Beschränkungen ein. Die Ausführungen hierzu sind zwar recht solide, es fragt sich indes, ob in einer Dissertation aus dem Jahre 2001 wirklich nochmals zwingend erklärt werden muss, was eine E-Mail oder eine Newsgroup ist. Überdies hätte Gets durch einen Blick in das einschlägige Schrifttum erkennen können, dass die für die rechtliche Bewertung wichtige technische Komplexität im Zusammenhang mit dem Internet weit über die in der Arbeit niedergelegten Bereiche hinausgeht. Das gilt auch für die möglichen Freiheitsbeschränkungen, die sich im Rahmen der Internet-Nutzung ergeben können (hierzu umfassend Germann, Gefahrenabwehr und Strafverfolgung im Internet, Berlin 2000).

Im zweiten Teil der Arbeit referiert die Autorin den bekannten und bereits vielfach aufgearbeiteten Diskussionsstand zum völkerrechtlichen Schutz der Meinungsäußerungs- und Informationsfreiheit. Dabei vertritt sie u. a. die Ansicht, dass das Prinzip des free flow of information keine völkerrechtliche Anerkennung gefunden habe (S. 59). Das wird mit einer einzigen Fundstelle belegt. Eine Auseinandersetzung mit den zahlreichen Autoren, die seit langer Zeit hierzu eine gegenteilige Auffassung vertreten, findet nicht statt. Ebenso wenig überzeugen kann der Hinweis darauf, dass die 
Allgemeine Menschenrechtserklärung aus dem Jahre 1948 keine gewohnheitsrechtliche Geltung habe, da es immer wieder zu Menschenrechtsverletzungen komme (S. 69). An dieser Stelle zeigt sich exemplarisch die an verschiedenen Stellen in dem Werk zu Tage tretende Problematik, dass Gets nicht abschließend mit dem aktuellen Stand der Dogmatik der Völkerrechtsquellen vertraut ist. Das mag auch daran liegen, dass oftmals auf fremdsprachliches Schrifttum verwiesen wird, das zumindest auf den ersten Blick noch eine gewisse Nähe zur sozialistischen Völkerrechtslehre aufweist. Überdies sei nochmals darauf hingewiesen, dass die wichtigen Gewährleistungen der EMRK zur aktiven und passiven Informationsfreiheit (S. 76-78) ohne jeden Verweis auf die Rechtsprechung des EuGHMR, die in diesem Bereich durch eine nicht unerhebliche Rechtsfortbildung geprägt ist, dargestellt werden.

Im dritten Teil der Arbeit geht es um die „Meinungs- und Informationsfreiheit und das Prinzip des freien Informationsflusses im Internet" (S. 97 ff.). Dabei wird zunächst noch einmal auf die einschlägigen völkervertraglichen Grundlagen der Meinungsäußerungsund Informationsfreiheit abgestellt, ohne dass freilich deutlich wird, wo der eigentliche Unterschied zur Darstellung im zweiten Teil der Arbeit besteht. Wenig überraschend ist, dass Gets eine völkergewohnheitsrechtliche Verankerung der genannten Freiheitsgewährleistungen ablehnt (S. 103-111). Näher belegt wird diese von der heute herrschenden Meinung wohl kaum geteilte Ansicht allerdings nicht. Der einzige diesbezügliche Verweis auf das Völkerrechtslehrbuch von Ipsen (S. 107/Fn. 458) geht fehl, da es in der zitierten Stelle bei Ipsen um die fremdenrechtlichen Mindeststandards und gerade nicht die allgemeinen Menschenrechte geht. Wenig verständlich ist auch die knappe Bemerkung auf S. 111, dass Staaten, die die Internetnutzung beschränken, nicht an ein Menschenrecht auf Meinungsäußerungsund Informationsfreiheit gebunden wären, wenn es sich in Zukunft herausbilden sollte. Gets bezieht sich hier vermutlich auf die so genannte Persistent-Objector-Regel, wobei deren Anwendbarkeit auf menschenrechtliche Regelungen allerdings überaus fraglich ist (vgl. nur Dahm/Delbrück/Wolfrum, Völkerrecht, Bd. I/3, 2. Aufl., Berlin u.a. 2002, S. 627).

Ab S. 135 stellt Gets noch die verschiedenen internationalen Rechtsinstrumentarien dar, die über den bislang diskutierten menschenrechtlichen Bereich hinausgehend für die Regulierung der elektronischen Information und Kommunikation wichtig sind. Dabei wird ein weiter Bogen von allgemeinen gesellschaftspolitischen Bestrebungen in der UNESCO bis zu spezifischen welthandelsrechtlichen Fragen im Rahmen der WTO-Rechtsordnung gespannt. Die spezifischen Besonderheiten und Problemlagen, die sich in den zahlreichen einschlägigen internationalen Rechtsordnungen stellen, werden indes nur oberflächlich und ohne umfängliche Berücksichtigung des einschlägigen Schrifttums abgehandelt. So muss es doch verwundern, dass die Autorin selbst einschlägige Habilitationsschriften nicht verarbeitet hat (z. B. zum WTO-Recht die grundlegende Arbeit von von Schorlemer, Globale Telekommunikation und Entwicklungsländer: die Liberalisierung von Telekommunikationsdiensten in GATT/WTO, Baden-Baden 2000).

Im Ergebnis ist festzuhalten, dass die Arbeit dem Anspruch ihres viel versprechenden Titels nicht gerecht $\mathrm{zu}$ werden vermag. Auch in $\mathrm{Zu}-$ kunft bleibt damit hinreichend Raum für weitere Forschung zum Themenkomplex „Völkerrecht und Internet".

Christian Tietje

\section{Michaela Tzankoff \\ Der Transformationsprozess in Bulgarien und die Entwicklung der postsozialistischen Medienlandschaft}

Hamburg: Lit, 2002. - 196 S.

ISBN 3-8258-5948-7

Der Zusammenbruch der kommunistischen Regime unter sowjetischer Vorherrschaft in Osteuropa und die Implementierung demokratischer Institutionen können in ihrer weltpolitischen Bedeutung kaum unterschätzt werden. Das Ende des Staatssozialismus bedeutete das Ende der Nachkriegsordnung und hat zu einer neuen Demokratisierungswelle weltweit geführt. Ungezählte Studien sind seitdem veröffentlicht worden, die den Umbruch und die Entwicklung der neuen Demokratien darstellen und zu erklären versuchen. In der Tat eröffnen sich faszinierende Forschungsfragen: Was ist Demokratie; unter welchen Bedingungen funktionieren demokratische Institutionen, oder auch nicht; welche Rolle spielen kulturel- 